PROCEEDINGS OF THE

AMERICAN MATHEMATICAL SOCIETY

Volume 139, Number 9, September 2011, Pages 3203-3216

S 0002-9939(2011)10756-1

Article electronically published on February 25, 2011

\title{
COUNTABLE RANDOM $p$-GROUPS WITH PRESCRIBED ULM-INVARIANTS
}

\author{
MANFRED DROSTE AND RÜDIGER GÖBEL
}

(Communicated by Birge Huisgen-Zimmermann)

\begin{abstract}
In this paper we present a probabilistic construction of countable abelian $p$-groups with prescribed Ulm-sequence. This result provides a different proof for the existence theorem of abelian $p$-groups with any given countable Ulm-sequence due to Ulm, which is sometimes called Zippin's theorem. The basic idea, applying probabilistic arguments, comes from a result by Erdős and Rényi. They gave an amazing probabilistic construction of countable graphs which, with probability 1, produces the universal homogeneous graph, therefore also called the random graph. P. J. Cameron says about this in his book Oligomorphic Permutation Groups [Cambridge University Press, 1990]: In 1963, Erdös and Rényi proved the following paradoxical result. . . . It is my contention that mathematics is unique among academic pursuits in that such an apparently outrageous claim can be made completely convincing by a short argument. The algebraic tool in the present paper needs methods developed in the 1970s, the theory of valuated abelian $p$-groups. Valuated abelian $p$-groups are natural generalizations of abelian $p$-groups with the height valuation, investigated in detail by F. Richman and E. Walker, and others. We have to establish extensions of finite valuated abelian $p$-groups dominated by a given Ulm-sequence. Probabilistic results of a similar nature have been established by A. Blass and G. Braun, and by M. Droste and D. Kuske.
\end{abstract}

\section{INTRODUCTION}

One of the most celebrated results in the theory of abelian groups is Ulm's theorem, which states that countable reduced $p$-groups are uniquely determined, up to isomorphism, by their Ulm sequences (Section 2); cf. Fuchs [13, Vol. 2, pp. 60, 61].

In a different setting, Fraïssé 12 and Jónsson 18 gave a model-theoretic method which implies, for instance, that there is a countable undirected graph $U$ which is universal, i.e. each countable graph embeds into $U$, and homogeneous, i.e. each isomorphism between two finite subgraphs of $U$ extends to an automorphism of $U$. Moreover, with these two properties, $U$ is determined uniquely up to isomorphism. Erdős and Rényi [10] gave a probabilistic construction of countable graphs which,

Received by the editors March 13, 2010 and, in revised form, July 9, 2010 and August 22, 2010 .

2000 Mathematics Subject Classification. Primary 20K10, 20K30; Secondary 60F20, 16W20.

Key words and phrases. Ulm's theorem, abelian p-groups, valuated group, amalgamation.

The authors are supported by the project No. 963-98.6/2007 of the German-Israeli Foundation for Scientific Research \& Development and by a project AOBJ 548025 of the Deutsche Forschungsgemeinschaft. 
amazingly, with probability 1 produces the universal homogeneous graph, therefore also called the random graph.

In view of this, the question arises whether a given countable reduced $p$-group can be viewed, in a suitable class of structures, as a universal and homogeneous object, thereby yielding its existence and uniqueness. Using the theory of valuated $p$-groups, as developed by Hunter, Richman, Walker (see e.g. [22]) in the 1970s, in [7. we showed that this is indeed the case.

Consequently, the question arises whether there is a probabilistic construction for countable valuated abelian $p$-groups with given Ulm-sequence. Indeed, several authors (e.g., [16, p. 352], 33, p. 352]) asked for an extension of Erdős and Renyi's probabilistic method to other structures than graphs.

It is the goal of this paper to give a probabilistic construction for countable valuated $p$-groups, with given Ulm-sequence $f$ as bound for the Ulm-invariants. We then show that with probability 1 , our construction produces a $p$-group $G$ realizing precisely the given sequence $f$ as its sequence of Ulm-invariants. We construct the groups $G$ with the set $\mathbb{N}$ of natural numbers as their domain. Moreover, we obtain $G$ as a union of a chain of finite valuated $p$-groups $A_{i}$ defined on the initial segments $\left\{0,1, \ldots, p^{i}-1\right\}(i \geq 0)$. The groups $A_{i}$ are constructed successively and probabilistically. In principle, this could be done by a machine; cf. also 21, [19] for constructive (non-probabilistic) versions of Ulm's theorem.

Algebraically, our main tool is a new extension result for enlarging a given finite valuated abelian $p$-group by one more generator realizing exactly a prescribed valuation. Related (but different) results were also developed in [17, 19]. As a consequence, we show that the class of finite abelian valuated $p$-groups satisfies the amalgamation property.

A related successive probabilistic construction of the countable universal homogeneous partially ordered set was given in [8]; see also Blass-Braun [2] for further interesting results in this direction. This procedure was extended to probabilistic constructions of universal causal sets [5] arising in theoretical physics and of universal Scott domains 8 and universal event structures [6] from theoretical computer science. We note that these objects are relational structures. The present valuated abelian $p$-groups seem to be the first functional structures with a probabilistic construction.

\section{Preliminaries on valuated abelian $p$-Groups}

We first collect the basics for our construction of reduced, abelian $p$-groups. We define the $\alpha$-th Ulm subgroups ( $\alpha$ an ordinal) inductively. Let $p^{0} G=G$ and $p^{\alpha} G=\bigcap_{\beta<\alpha} p\left(p^{\beta} G\right)$. The length of a reduced $p$-group $G$ is the minimal ordinal $\lambda$ with $p^{\lambda} G=0$. The definition of the $p$-height $\alpha$ of an element $x \in G$, denoted by $h_{p}(x)=\alpha$, is an immediate consequence of the definition of Ulm subgroups: the $p$-height of $x$ is the unique ordinal $\alpha<\lambda$ with $x \in p^{\alpha} G \backslash p^{\alpha+1} G$. Finally, the Ulm-invariants are obtained by the homomorphisms

$$
\mu: p^{\alpha} G / p^{\alpha+1} G \longrightarrow p^{\alpha+1} G / p^{\alpha+2} G \text { which are induced by multiplication by } p \text {. }
$$

It is clear that the kernel of $\mu$ is a subspace $U_{G}(\alpha)$ of the vector space $\left(p^{\alpha} G / p^{\alpha+1} G\right)[p]$. This vector space (or its dimension over $Z_{p}$ ) is also denoted by $f_{G}(\alpha)$, and is the $\alpha$-th Ulm-invariant. If $G$ is countable, then the Ulm-sequence 
$f(\alpha)_{\alpha<\lambda}=\left(f_{G}(\alpha)\right)_{\alpha<\lambda}$ must satisfy the following conditions (see [13, Vol. 1, $(37.6)])$.

(i) There is a countable (minimal) ordinal $\lambda$ (the length $\lambda=\lambda(f)$ ) with $f(\alpha)=$ 0 for all $\alpha \geq \lambda$.

(ii) $\operatorname{dim} f(\alpha) \leq \aleph_{0}$ for all $\alpha<\lambda$.

(iii) If $\alpha<\lambda$, then there is $n \in \mathbb{N}_{0}$ with $f(\alpha+n) \neq 0$.

Since we are only interested in countable abelian groups, we call a mapping $f$ satisfying these conditions a U-sequence. It follows that for each U-sequence $f$ there is a $p$-group $G$ with $f=f_{G}$, which is the existence part of Ulm's theorem from [23] (often named after Zippin because of 24]; cf. [14] for historical remarks).

There is a natural extension of these notions and results to valuated abelian $p$-groups, derived in the 1970s and mainly due to Richman and Walker 22. (but see also the references in this fundamental paper). This will be an important tool for our paper.

A valuated $p$-group is an abelian $p$-group $G$ with a valuation map $v: G \longrightarrow$ $\lambda \cup\{\infty\}$ into a large enough ordinal $\lambda$ adjoining the symbol $\infty$ such that the following holds for all $x, y \in G$ :

(a) $v(p x)>v(x)$,

(b) $v(x+y) \geq \min (v(x), v(y))$,

(c) $v(n x)=v(x)$ if $p$ does not divide $n$.

The symbol $\infty$ satisfies $\infty<\infty$ and $\alpha<\infty$ for any ordinal $\alpha<\lambda$. Thus $v(0)=\infty$ and the valuated $p$-group $(G, v)$ is called reduced if $v^{-1}(\infty)=\{0\}$. We will often use the fact that if $x, y \in G$ with $v(x) \neq v(y)$, then $v(x+y)=\min \{v(x), v(y)\}$.

Moreover, $G(\alpha)=\{g \in G \mid v(x) \geq \alpha\}$ extends the notion of the $\alpha$-th Ulm subgroup to valuated $p$-groups for any ordinal $\alpha$, and a morphism of valuated groups $\eta:(G, v) \longrightarrow(H, v)$ is a group homomorphism $\eta: G \longrightarrow H$ with $v(x \eta) \geq v(x)$ for all $x \in G$.

If $\left(A, v_{A}\right),(B, v)$ are two valuated groups, then $\left(A, v_{A}\right) \subseteq(B, v)$ as valuated groups if $A \subseteq B$ are subgroups and $v_{A}=v\lceil A$. From an ascending chain of valuated groups $\left\{\left(A_{i}, v_{i}\right) \mid i<\nu\right\}$ we get a valuated union $\bigcup_{i<\nu}\left(A_{i}, v_{i}\right)=\left(\bigcup_{i<\nu} A_{i}, \bigcup_{i<\nu} v_{i}\right)$.

The most important valuation for abelian $p$-groups is the classical heightvaluation mentioned above. In fact a theorem by Richman and Walker [22, p. 161, Theorem 23] says that any valuation is induced by a height valuation; i.e., for every valuated $p$-group $(G, v)$ there is a group $H$ with $G \subseteq H$ and height valuation $h_{p}$ such that $v=h_{p}\lceil G$. The $p$-height-function on $G$ is characterized (cf. 21, Theorem, p. 74]) by

$h_{p}(x)=\sup \left\{h_{p}(y)+1 \mid x=p y\right\}$, where $h_{p}(x)$ is either an ordinal number or $\infty$.

The valuated Ulm subgroups $G(\alpha), G(\alpha+1)$ and $G(\alpha+2)$ are used to define the Ulm-invariants $f_{G, v}(\alpha)$ of the valuated $p$-group $(G, v)$ :

$$
f_{G, v}(\alpha)=\frac{\langle x \in G(\alpha) \mid p x \in G(\alpha+2)\rangle}{G(\alpha+1)} .
$$

Hence we have a map $f_{G, v}: \lambda+1 \longrightarrow Z_{p}-\operatorname{Mod}$ (the category of $Z_{p}$-vector spaces), which is called the Ulm-sequence of the valuated group $(G, v)$ (for short U-sequence). A U-sequence $f=f_{G, v}$ of a reduced valuated countable abelian $p$ group (these are the only ones we are interested in) must also satisfy the conditions (i),(ii),(iii) above; see Richman [20, p. 632] or [19, p. 282, Theorem 5.10]. 
Moreover, if $f, g$ are two Ulm-sequences, then $f \leq g$ if $f(\alpha) \leq g(\alpha)$ (equivalently $f(\alpha)$ embeds as a subspace into $g(\alpha))$ for all $\alpha \in \operatorname{Dom} f$. If $(A, v)=\bigcup_{i<\nu}\left(A_{i}, v_{i}\right)$ is the union of a chain of valuated groups, then $f_{A, v}=\bigcup_{i<\nu} f_{A_{i}, v_{i}}$ holds naturally.

\section{Extensions OF VALUATED ABELIAN $p$-GROUPS}

In this section, we will investigate extensions of finite valuated abelian groups with a given U-sequence as the bound for their Ulm-invariants. First we will consider 'small' extensions.

Definition 3.1. Let $f$ be a U-sequence. We will denote extensions of valuated abelian groups of the form $(H, v) \subseteq(K, v)$ with $K=\langle H, z\rangle, p z \in H$ and Ulmsequences $f_{H, v} \leq f_{K, v} \leq f$ (as in the next lemma) as one-point $f$-extensions.

The next lemma resembles Lemma 8 from [17, p. 355] and Lemma 5.2 from [19, p. 282], but because of the required upper bound $f$ for the Ulm-sequence, the claim and also its proof are quite different. It is algebraically the main ingredient for our probabilistic Theorem 4.1

Extension Lemma 3.2. Let $f$ be a U-sequence such that either its length $\lambda$ is a limit ordinal or $\lambda=\rho+1$ with $f(\rho)=\aleph_{0}$. Moreover suppose $(H, v)$ is a finite, reduced, valuated abelian p-group with $f_{H, v} \leq f$. Let $a \in H, \alpha<v(a)$ and assume that $f(\alpha) \neq 0$ if $v(a) \geq \alpha+2$.

Then there are a valuated abelian p-group $\left(K, v^{\prime}\right) \supseteq(H, v)$ and $z \in K$ such that $f_{K, v^{\prime}} \leq f, K=\langle H, z\rangle, p z=a$ and $v^{\prime}(z)=\alpha$. Moreover, if $A \subseteq H$ is given with $f_{A, v}(\alpha)<f_{H, v}(\alpha)$, we can construct $v^{\prime}$ such that $v^{\prime}(x+m z)=\min \{v(x), \alpha\}$ for each $x \in A$ and $0<m<p$.

Proof. We define $K$ naturally by adjoining an element $z$ to $H$ (formally by a pushout) subject only to the relation $p z=a$. In order to define the valuation $v^{\prime}$ on $K$, we introduce two cases:

- The critical case: $f_{H, v}(\alpha)=f(\alpha)$ and either $v(a) \geq \alpha+2$ or there is $x \in H$ with $v(x)=\alpha$ and $v(p x+a) \geq \alpha+2$.

- The non-critical case: $f_{H, v}(\alpha)<f(\alpha)$ or both $v(a)=\alpha+1$ and $v(p x+a)=$ $\alpha+1$ for each $x \in H$ with $v(x)=\alpha$.

Note that if $x \in H$ and $v(x)=\alpha$, then by assumption of the lemma, $v(a) \geq \alpha+1$ and thus $v(p x+a) \geq \min \{v(p x), v(a)\} \geq \alpha+1$, so the two cases are indeed complementary.

We first deal with the non-critical case. We let $v^{\prime} \mid H=v$, and note that elements of $K$ are of the form $x+m z$ for $x \in H$ and $0 \leq m<p$. We put $v^{\prime}(x+m z)=\min \{v(x), \alpha\}$ for each $x \in H$ and $0<m<p$. Clearly $v^{\prime}$ satisfies the three axioms (a),(b),(c) (of Section 2) and thus $v^{\prime}$ is a valuation on $K$. We claim that $f_{K, v^{\prime}} \leq f$.

Let $x \in H, 0<m<p$ and $\beta=v^{\prime}(x+m z)$.

If $\beta=v(x)<\alpha$, we have $x+m z \equiv x \bmod K(\beta+1)$ by $v^{\prime}(m z)=\alpha \geq \beta+1$. So, $x+m z$ does not increase $f_{K, v^{\prime}}(\beta)$ in comparison to $f_{H, v}(\beta)$; see (2.2).

Now let $\beta=\alpha$. If $f_{H, v}(\alpha)<f(\alpha)$, then clearly $f_{K, v^{\prime}}(\alpha) \leq f_{H, v}(\alpha)+1 \leq f(\alpha)$ as needed. Now let $f_{H, v}(\alpha)=f(\alpha)$. Then $v(a)=\alpha+1$ by assumption of the non-critical case. 
If $v(x)>\alpha$, we have $v(p x)>\alpha+1$, so $v(p(x+m z))=v(p x+m a)=\alpha+1$, which also holds for $x=0$ and $m=1$. Hence neither $z$ nor $x+m z$ increases $f_{K, v^{\prime}}(\alpha)$ in comparison to $f_{H, v}(\alpha)$.

Finally let $v(x)=\alpha$. Since $0<m<p$, we have $v(p(x+m z))=v(p x+m a)=$ $v(p \bar{x}+a)$ for some $\bar{x} \in H$ with $v(\bar{x})=v(x)=\alpha$. So $v(p \bar{x}+a)=\alpha+1$ by assumption of the non-critical case, and again neither $z$ nor $x+m z$ increases $f_{K, v^{\prime}}(\alpha)$ versus $f_{H, v}(\alpha)$. Hence $f_{K, v^{\prime}} \leq f$.

Now we consider the critical case. We assume that in case $f_{H, v}(\alpha) \neq 0$ we are also given a valuated subgroup $A \subseteq H$ with $f_{A, v}(\alpha)<f_{H, v}(\alpha)$ (possibly $(A, v)=$ $(\{0\},\{(0, \infty)\})$.

First we wish to find and fix an element

(3.1) $x^{\prime} \in H$ with $v\left(x^{\prime}\right)=\alpha, v\left(p x^{\prime}+a\right) \geq \alpha+2$, and $x^{\prime} \notin A$ if $f_{A, v}(\alpha)<f_{H, v}(\alpha)$.

Indeed first assume $f_{A, v}(\alpha)<f_{H, v}(\alpha)$. Then by (2.2) there is $x_{1} \in H \backslash A$ with $v\left(x_{1}\right)=\alpha$ and $v\left(p x_{1}\right) \geq \alpha+2$.

If $v(a) \geq \alpha+2$, we put $x^{\prime}=x_{1}$ and note that $v\left(p x^{\prime}+a\right) \geq \min \left\{v\left(p x^{\prime}\right), v(a)\right\} \geq$ $\alpha+2$.

Now let $v(a)=\alpha+1$. By assumption of the critical case, there is $x_{2} \in H$ with $v\left(x_{2}\right)=\alpha$ and $v\left(p x_{2}+a\right) \geq \alpha+2$. If $x_{2} \notin A$, we put $x^{\prime}=x_{2}$. If $x_{2} \in A$, we put $x^{\prime}=x_{1}+x_{2}$. Then $x^{\prime} \in H \backslash A$. Since $v(a)=\alpha+1$ and $v\left(p x_{2}+a\right) \geq \alpha+2$, we obtain $v\left(p x_{2}\right)=\alpha+1$. Hence $v\left(p x^{\prime}\right)=v\left(p x_{1}+p x_{2}\right)=\alpha+1$ by $v\left(p x_{1}\right) \geq \alpha+2$. So $\alpha+1=v\left(p x^{\prime}\right)>v\left(x^{\prime}\right) \geq \min \left\{v\left(x_{1}\right), v\left(x_{2}\right)\right\}=\alpha$ implies $v\left(x^{\prime}\right)=\alpha$. Then $v\left(p x^{\prime}+a\right)=v\left(p x_{1}+\left(p x_{2}+a\right)\right) \geq \alpha+2$.

Finally, assume $f_{H, v}(\alpha)=0$. By the assumption of the critical case and the lemma, then $f(\alpha)=0, v(a)=\alpha+1$ and there is $x^{\prime} \in H$ with $v\left(x^{\prime}\right)=\alpha$ and $v\left(p x^{\prime}+a\right) \geq \alpha+2$, as needed. Hence (3.1) follows.

- Next we define the valuation $v^{\prime}$ on $K$.

Let $v^{\prime} \uparrow H=v$. We use the element $x^{\prime}$ from (3.1) to define $v^{\prime}$ on $K \backslash H$. If $x \in H, 0<m<p$ and $x \neq m x^{\prime}$, we put $v^{\prime}(x+m z)=\min \{v(x), \alpha\}$. Note that in case $x^{\prime} \notin A$, we also have $m x^{\prime} \notin A$, so in this case we have in particular $v^{\prime}(x+m z)=\min \{v(x), \alpha\}$ for each $x \in A$ (from $\left.x \neq m x^{\prime}\right)$.

Next assume that $x=m x^{\prime}$. Then $v(p x+m a)=v\left(p x^{\prime}+a\right) \geq \alpha+2$ by the choice of $x^{\prime}$ and because multiplication by $m$ does not change the valuation.

We distinguish between three cases.

Case A. Let $v\left(p x^{\prime}+a\right)=\gamma+1$ be a successor ordinal.

Then we put $v^{\prime}(x+m z)=v^{\prime}\left(x^{\prime}+z\right)=\gamma$. From $v\left(p x^{\prime}+a\right) \geq \alpha+2$ follows $\gamma \geq \alpha+1$

Case B. Let either $v\left(p x^{\prime}+a\right)$ be a limit ordinal and $p x^{\prime}+a \neq 0$ or let $p x^{\prime}+a=0$ and the length $\lambda$ of $f$ also be a limit.

Then we may now choose $0<n \in \mathbb{N}$ such that $\alpha+n \notin v(H)$ (a finite set) and $f(\alpha+n) \neq 0$ (a property of U-sequences). We put $v^{\prime}(x+m z)=v^{\prime}\left(x^{\prime}+z\right)=\alpha+n \geq$ $\alpha+1$. Observe that $\alpha+n<v\left(p x^{\prime}+a\right)$ and $f_{H, v}(\alpha+n)=0$.

Case C. Let $p x^{\prime}+a=0$ and $\lambda=\rho+1$ be a successor ordinal. Then $f(\rho)=\aleph_{0}$ by the hypothesis of the lemma. 
In this case we let $v^{\prime}(x+m z)=v^{\prime}\left(x^{\prime}+z\right)=\rho$. Trivially, $\rho<v\left(p x^{\prime}+a\right)=\infty$.

It is straightforward to check that $v^{\prime}$ is a valuation on $K$, and it remains to show that $f_{K, v^{\prime}} \leq f$ in these cases. Let $x \in H, 0<m<p$ and $\beta=v^{\prime}(x+m z)$.

If $\beta<\alpha$, we have $v(x)=\beta$ and $x+m z \equiv x \bmod K(\beta+1)$ by $v^{\prime}(m z)=\alpha \geq \beta+1$. So, $x+m z$ does not increase $f_{K, v^{\prime}}(\beta)$ in comparison to $f_{H, v}(\beta)$; see again (2.2).

If $\beta>\alpha$, we have $x=m x^{\prime}$. In Case $\mathrm{\AA}$, we have $\beta=\gamma$. Then $v^{\prime}(p(x+m z))=$ $v^{\prime}\left(p x^{\prime}+a\right)=\gamma+1$, so $x+m z$ does not increase $f_{K, v^{\prime}}(\beta)$ in comparison to $f_{H, v}(\beta)$; check (2.2). In Case B we have $\beta=\alpha+n$ and so $f_{K, v^{\prime}}(\beta) \leq f_{H, v}(\beta)+1=1 \leq f(\beta)$. In Case C we have $\beta=\rho$ and $f_{K, v^{\prime}}(\rho) \leq \aleph_{0}=f(\rho)$.

Now let $\beta=\alpha$. Note that $v^{\prime}(z)=\alpha$ and if $v(a) \geq \alpha+2$, then $z$ could increase $f_{K, v^{\prime}}(\alpha)$ in comparison to $f_{H, v}(\alpha)$. Likewise, if $v(p x+m a) \geq \alpha+2$, the element $x+m z$ could contribute to such an increase. But here we have $v\left(p x^{\prime}+a\right) \geq \alpha+2$, so in Cases A and B, and if $\rho>\alpha$ also in Case C], we obtain $v^{\prime}\left(x^{\prime}+z\right) \geq \alpha+1$ and $x^{\prime}+z \in K(\alpha+1) \backslash H$. In Case $\mathrm{C}$ with $\rho=\alpha$ we have $f_{K, v^{\prime}}(\alpha) \leq \aleph_{0}=f(\alpha)$ by assumption of the lemma. Hence, in these cases $f_{K, v^{\prime}}(\alpha)=f_{H, v}(\alpha)=f(\alpha)$ as needed. Thus $f_{K, v^{\prime}} \leq f$.

The referee kindly pointed out the following example (now) showing that the assumptions for Lemma 3.2 are also necessary. Take $f$ to be a U-sequence of length $\omega+1$ with $f(n)=1$ for all $n<\omega$ and $f(\omega)=1$. Moreover, let $H=\langle x\rangle$ be the cyclic group of order $p$ with valuation $v(x)=\omega$ and take $a=0$ in $H$ and $\alpha=\omega$. Then the extension $\left(K, v^{\prime}\right) \supseteq(H, v)$ in the lemma does not exist.

For later use we note that the assumption of Lemma 3.2 on the length $\lambda$ of $f$ was used only in Case $\mathrm{C}$ of the proof above. Now we can show that the finite valuated $p$-groups with given bound for their Ulm-invariants satisfy the amalgamation property.

Amalgamation Lemma 3.3. Let $f$ be a U-sequence and $(A, v),(B, v)$ and $(H, v)$ be finite, reduced, valuated abelian p-groups such that $(A, v) \subseteq(B, v),(A, v) \subseteq$ $(H, v), A=B \cap H$ and $f_{B, v}, f_{H, v} \leq f$. Then there is a finite, valuated abelian p-group $\left(K, v^{\prime}\right)$ such that $f_{K, v^{\prime}} \leq f,(H, v) \subseteq\left(K, v^{\prime}\right)$, and there is a valuated embedding $\varphi:(B, v) \hookrightarrow\left(K, v^{\prime}\right)$ with $\varphi \mid A=\operatorname{id}_{A}$. Thus we get the following diagram:

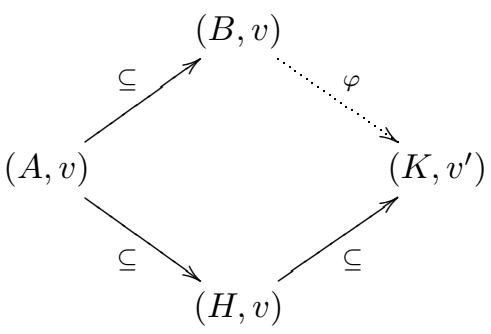

Proof. We may assume that $B=\langle A, y\rangle$ for some $y \in B \backslash A$ with $p y \in A$. We choose an element $y \in B \backslash A$ such that $v(y)=\sup (v(B \backslash A)$ ) (this is to say that $y$ is a proper element; see [13]) and furthermore, $v(p y) \geq v\left(p y^{\prime}\right)$ for each $y^{\prime} \in B \backslash A$ with $v(y)=v\left(y^{\prime}\right)$. Put $a=p y$ and $\alpha=v(y)$. Note that $v(a)=v(p y)>v(y)$ and $a \in A$. Also $B(\alpha+1)=A(\alpha+1)$ due to the maximality of $v(y)=\alpha$. Hence, if $v(p y)=v(a) \geq \alpha+2$, then by $y \in B(\alpha) \backslash A$ we obtain $f_{A}(\alpha)<f_{B}(\alpha) \leq f(\alpha)$, so $f(\alpha) \neq 0$, realizing an assumption of Lemma 3.2 . 
We claim that $v(x+m y)=\min \{v(x), \alpha\}$ for all $0<m<p$. Indeed, if $v(x) \neq \alpha$, this is clear. If $v(x)=\alpha$, we have $v(x+m y) \geq \alpha$ and so $v(x+m y)=\alpha$ by our assumption on $y$, proving our claim.

Subsequently, we will either find a valuated embedding $\varphi: B \longrightarrow H$ or we construct an extension $\left(K, v^{\prime}\right) \supseteq(H, v)$ as in the Extension Lemma 3.2 and a valuated embedding $\varphi: B \longrightarrow K$, depending on several cases.

We show that here we will not need the assumption of Lemma 3.2 on the length $\lambda$ of $f$.

First we assume that the quadruple $(H, v, a, \alpha)$ realizes the non-critical case of the proof of Lemma3.2. Then we construct $\left(K, v^{\prime}\right)$ with $K=\langle H, z\rangle, z \in K \backslash H$ and $p z=a$ as in the proof of Lemma 3.2. Then $f_{K, v^{\prime}} \leq f$, and we define $\varphi: B \longrightarrow K$ by $(x+m y) \varphi=x+m z$ for all $x \in A$ and $0 \leq m<p$. Since $p y=a=p z$, clearly $\varphi$ is a group embedding with $\varphi\left\lceil A=\mathrm{id}_{A}\right.$ and $y \varphi=z$. As noted above, resp. by definition of $v^{\prime}$, we have

$$
v(x+m y)=\min \{v(x), \alpha\}=v^{\prime}(x+m z) \text { for each } x \in A \text { and } 0<m<p .
$$

Hence $\varphi$ preserves the valuation.

Therefore from now on we assume that $(H, v, a, \alpha)$ satisfies the conditions of the critical case of the proof of Lemma 3.2. For later use we note the following condition (3.2).

Assume there is an element $x^{\prime} \in H \backslash A$ with $v\left(x^{\prime}\right)=\alpha, p x^{\prime}+a=0$

$$
\text { and } v\left(\bar{a}+m\left(-x^{\prime}\right)\right)=\alpha \text { for each } \bar{a} \in A \text { with } v(\bar{a})=\alpha \text { and each } 0<m<p \text {. }
$$

If condition (3.2) is satisfied, we define $\varphi: B \longrightarrow H$ by letting $(\bar{a}+m y) \varphi=$ $\bar{a}+m\left(-x^{\prime}\right)$ for each $\bar{a} \in A$ and $0 \leq m<p$. Since $p y=a=p\left(-x^{\prime}\right), \varphi$ is a homomorphism and $\varphi$ is injective since $x^{\prime} \notin A$. We note that $v(\bar{a}+m y)=$ $\min \{v(\bar{a}), \alpha\}=v^{\prime}\left(\bar{a}+m\left(-x^{\prime}\right)\right)$ for each $\bar{a} \in A$ and $0<m<p$ : If $v(\bar{a}) \neq \alpha$, the second equality is clear by $v\left(x^{\prime}\right)=\alpha$, and if $v(\bar{a})=\alpha$ it is satisfied by the assumption of condition (3.2). Hence $\varphi$ is a valuated embedding with $\varphi\left\lceil A=\operatorname{id}_{A}\right.$, as needed.

Now we distinguish between two cases.

Case 1. Let $f_{A, v}(\alpha)=f_{B, v}(\alpha)$.

Since we are in the critical case, as shown in the proof of Lemma 3.2, we can find a fixed element $x^{\prime} \in H$ with $v\left(x^{\prime}\right)=\alpha$ and $v\left(p x^{\prime}+a\right) \geq \alpha+2$.

Suppose we had $x^{\prime} \in A$. Then $x^{\prime}+y \in B \backslash A$ and $v\left(x^{\prime}+y\right)=\alpha$ by our choice of $y$. Moreover, we have $v\left(p\left(x^{\prime}+y\right)\right)=v\left(p x^{\prime}+a\right) \geq \alpha+2$, and $B(\alpha+1)=A(\alpha+1)$ by our choice of $y$ with maximality of $v(y)=\alpha$. But this implies $f_{A, v}(\alpha)<f_{B, v}(\alpha)$, contradicting the assumption of this case. Thus $x^{\prime} \notin A$.

First assume $p x^{\prime}+a=0$. Suppose there are $\bar{a} \in A$ and $0<m<p$ such that $v(\bar{a})=\alpha$ and $v\left(\bar{a}+m\left(-x^{\prime}\right)\right) \geq \alpha+1$. Then $\bar{a}+m y \in B \backslash A$ has value $v(\bar{a}+m y)=\alpha$ and $v(p(\bar{a}+m y))=v(p \bar{a}+m a)=v\left(p\left(\bar{a}+m\left(-x^{\prime}\right)\right)\right) \geq \alpha+2$. Using $B(\alpha+1)=A(\alpha+1)$, it follows that $f_{A, v}(\alpha)<f_{B, v}(\alpha)$, contradicting the assumption of this case. Hence for all $\bar{a} \in A$ with $v(\bar{a})=\alpha$ and $0<m<p$ we have $v\left(\bar{a}+m\left(-x^{\prime}\right)\right)=\alpha$. So condition (3.2) is satisfied, and we obtain a valuated embedding $\varphi: B \longrightarrow H$ as noted above.

Secondly assume that $p x^{\prime}+a \neq 0$. Then we construct $K=\langle H, z\rangle$ with $p z=$ $a \in A$ and the valuation $v^{\prime}$ on $K$ using the element $x^{\prime}$ as described in the proof 
of Lemma 3.2. Note that by our assumption $p x^{\prime}+a \neq 0$, Case $\mathrm{C}$ cannot occur, and we obtain $f_{K, v^{\prime}} \leq f$. We define $\varphi: B \longrightarrow K$ by letting $(x+m y) \varphi=x+m z$ for each $x \in A$ and $0 \leq m<p$. Since $p y=a=p z$ and $z \notin H, \varphi$ is a group embedding. As noted above and by $x^{\prime} \notin A$ and the construction of $v^{\prime}$ we have $v(x+m y)=\min \{v(x), \alpha\}=v^{\prime}(x+m z)$ for each $x \in A$ and $0<m<p$. Hence $\varphi: B \longrightarrow K$ is a valuated embedding with $\varphi \uparrow A=\mathrm{id}_{A}$, as needed.

Case 2. Let $f_{A, v}(\alpha)<f_{B, v}(\alpha)$.

By assumption of the critical case, we have $f_{H, v}(\alpha)=f(\alpha)$. Hence $f_{A, v}(\alpha)<$ $f_{H, v}(\alpha)$. Consequently, there is $x^{\prime} \in H \backslash A$ with $v\left(x^{\prime}\right)=\alpha$ and $v\left(p x^{\prime}\right) \geq \alpha+2$ and $v\left(\bar{a}+m\left(-x^{\prime}\right)\right) \leq \alpha$ for all $\bar{a} \in A$ and $0<m<p$. For if we had $v\left(\bar{a}+m\left(-x^{\prime}\right)\right) \geq \alpha+1$ for some $\bar{a} \in A$ and $0<m<p$, then $x^{\prime}$ could not contribute to $f_{A, v}(\alpha)<f_{H, v}(\alpha)$. Consequently, in the case that $p x^{\prime}+a=0$, condition (3.2) is satisfied and we obtain a valuated embedding $\varphi: B \longrightarrow H$ as noted above.

Therefore we may assume that $p x^{\prime}+a \neq 0$. Suppose we had $v(a)=\alpha+1$. If $x \in B(\alpha) \backslash A$, then $v(x)=\alpha$ by the maximality of $v(y)=\alpha$, and by the choice of $y$ and $a=p y$, it follows that $v(p x) \leq v(p y)=v(a)=\alpha+1$, so $v(p x)=\alpha+1$. Hence $f_{A, v}(\alpha)=f_{B, v}(\alpha)$, which contradicts the assumption of this case.

Hence $v(a) \geq \alpha+2$ and thus also $v\left(p x^{\prime}+a\right) \geq \alpha+2$. Now we construct $K=\langle H, z\rangle$ with $p z=a$ and the valuation $v^{\prime}$ on $K$ using the element $x^{\prime}$, as described in the proof of Lemma 3.2. Note that since $p x^{\prime}+a \neq 0$, Case C cannot occur and we obtain $f_{K, v^{\prime}} \leq f$. As in Case 1 by $x^{\prime} \notin A$ the mapping $\varphi: B \longrightarrow K$ with $(x+m y) \varphi=x+m z$ for each $x \in A$ and $0 \leq m<p$ is a valuated embedding with $\varphi\left\lceil A=\operatorname{id}_{A}\right.$.

The Amalgamation Lemma 3.3 fixes a gap in the proof of the corresponding result in [7] (where, on p. 59, Case 2, the element $a^{\prime}$ in general only satisfies $v\left(a^{\prime}\right) \geq \beta$, instead of $\left.v\left(a^{\prime}\right)=\beta\right)$.

Let $f$ be a $\mathrm{U}$-sequence. We say that a valuated group $(G, v)$ realizes all one-point $f$-extensions of finite subgroups if whenever $(A, v) \subseteq(G, v)$ is a finite subgroup and $(A, v) \subseteq\left(B, v^{\prime}\right)$ is a one-point $f$-extension, there is an embedding $\varphi:\left(B, v^{\prime}\right) \hookrightarrow$ $(G, v)$ with $\varphi \uparrow A=\operatorname{id}_{A}$.

In order to state the connections between our result and [7] we recall some notation from 7 .

Let $\mathfrak{C}_{f}=\left\{(H, v) \mid f_{H, v} \leq f\right\}$. We call a valuated group $(G, v) \in \mathfrak{C}_{f}$

- universal for $\mathfrak{C}_{f}$, if each $\left(H, v^{\prime}\right) \in \mathfrak{C}_{f}$ embeds into $(G, v)$,

- homogeneous if each isomorphism between two finite valuated subgroups of $(G, v)$ extends to an automorphism of $(G, v)$.

It follows from standard back-and-forth arguments (cf. 7]) that a group $(G, v) \in \mathfrak{C}_{f}$ is homogeneous and universal for $\mathfrak{C}_{f}$ if and only if $(G, v)$ realizes all one-point $f$-extensions of finite subgroups, and any two such objects are isomorphic.

Now we show

Lemma 3.4. Let $f$ be a $U$-sequence and $(G, v)$ a valuated abelian p-group such that $f_{G, v} \leq f$. Assume that $(G, v)$ realizes all one-point $f$-extensions of finite subgroups. Then $v=h_{G}$ is the height valuation of $G$, and $f_{G}=f$.

Proof. This was shown in Droste and Göbel [7, pp. 59-60], but we include the argument for the convenience of the reader. First we claim

$$
\text { If } a \in G \text { and } \alpha<v(a) \text {, then } a=p y \text { for some } y \in G \text { and } v(y) \geq \alpha \text {. }
$$


Indeed, consider $(\langle a\rangle, v) \subseteq(G, v)$. By [19, Lemma 5.2] we can find a one-point $f$-extension $(\langle a\rangle, v) \subseteq\left(K, v^{\prime}\right)$ and $y^{\prime} \in K$ with $p y^{\prime}=a$ and $v^{\prime}\left(y^{\prime}\right) \geq \alpha$. By assumption, there is an embedding $\varphi:\left(K, v^{\prime}\right) \hookrightarrow(G, v)$ with $\varphi\lceil\langle a\rangle=$ id. Hence $y=y^{\prime} \varphi \in G$ satisfies $a=p y$ and $v(y)=v^{\prime}\left(y^{\prime}\right) \geq \alpha$. Now (3.3) and (2.1) imply $v=h_{G}$.

It remains to show that $f_{G}=f$. By assumption and the above, $f_{G}=f_{G, v} \leq$ $f$. Choose any $\alpha<\lambda$ and any $Z_{p}$-vector space $V$ of dimension $f(\alpha)$. We write $V=\bigcup_{i \in \mathbb{N}_{0}, i \leq f(\alpha)} V_{i}$ with $V_{i} \subseteq V_{i+1}$ and $\operatorname{dim} V_{i}=i$ for each $i \leq f(\alpha)$. This turns into a valuated group $\left(V, v^{\prime}\right)$ by letting $v^{\prime}(x)=\alpha$ for each $x \in V \backslash\{0\}$. Then $\left(V_{i}, v^{\prime}\right) \subseteq\left(V_{i+1}, v^{\prime}\right)$ is a one-point $f$-extension. By our assumption, we can successively embed the $\left(V_{i}, v^{\prime}\right)$ into $(G, v)$, obtaining an embedding of $\left(V, v^{\prime}\right)$ into $(G, v)$. Hence $f(\alpha)=f_{V, v^{\prime}}(\alpha) \leq f_{G, v}(\alpha) \leq f(\alpha)$, showing $f_{G}(\alpha)=f(\alpha)$.

In order to prove the existence part of Ulm's theorem, it therefore remains to construct a group $(G, v) \in \mathfrak{C}_{f}$ which realizes all one-point $f$-extensions of finite subgroups. Since the finite valuated groups in $\mathfrak{C}_{f}$ by Lemma 3.3 satisfy the amalgamation property, it follows from a category-theoretic version of the Fraïssé-Jónsson theorem from model theory that there is such an object $(G, v) \in \mathfrak{C}_{f}$ as required; cf. [7. In the sequel we will give a probabilistic construction of groups $(G, v) \in \mathfrak{C}_{f}$.

\section{The probability for Realizing Given Ulm-SEQuences}

4.1. The construction. Let $f$ be a countable Ulm-sequence of length $\lambda=\lambda(f)$.

If $f$ has finite length and is the Ulm-sequence of an abelian $p$-group $G$, then $G$ is bounded and thus a direct sum of cyclic groups by the Baer-Prüfer Theorem (see [13, p. 88, Theorem 17.2]). Hence the characterization of $G$ is obvious. By Definition 2.2 (for $v=h_{p}$ ) $G$ is a finite direct sum of homogeneous summands $G_{\alpha}=Z_{\alpha}^{(f(\alpha))}$ with the convention that $G_{\alpha}=0$ if $f(\alpha)=0$.

Thus we may assume that $\lambda \geq \omega$. We will construct a reduced abelian $p$-group $(G,+)$ with domain $G=\mathbb{N}_{0}$ and Ulm-invariant $f_{G} \leq f$. For $\kappa=\infty$ and for each ordinal $\kappa<\lambda$, we let $I(\kappa)=\{\alpha<\kappa \mid \alpha$ an ordinal, $f(\alpha) \neq 0$ or $\kappa=\alpha+1\}$, and we assume that we are given a discrete probability measure $\mu_{\kappa}$ on $I(\kappa)$, i.e. $\sum_{\alpha \in I(\kappa)} \mu_{\kappa}(\alpha)=1$ and $\mu_{\kappa}(\alpha) \neq 0$ for each $\alpha \in I(\kappa)$. We will obtain $(G,+)$ as the union of a chain $\left(A_{i}\right)_{i \in \mathbb{N}_{0}}$ of finite valuated abelian $p$-groups with $A_{i}=\left\{0, \ldots, p^{i}-1\right\}$ (thus $\left.\left|A_{i}\right|=p^{i}\right)$ and $\left(A_{i}, v_{i}\right) \subseteq\left(A_{i+1}, v_{i+1}\right)$ as valuated subgroups for each $i \in \mathbb{N}_{0}$. We proceed as follows.

Let $A_{0}=\{0\}$. For each $i \in \mathbb{N}_{0}$, let $p_{i}=p^{i}$. Now let $i \in \mathbb{N}_{0}$ and assume the group $\left(A_{i},+, v_{i}\right)$ with valuation $v_{i}$ has been constructed. We will construct the group $\left(A_{i+1},+, v_{i+1}\right)$ with $A_{i+1}=\left\langle A_{i}, p_{i}\right\rangle$ such that $A_{i+1}$ has universe $\left\{0, \ldots, p^{i+1}-1\right\}$ and $p p_{i} \in A_{i}$ in four probabilistic steps. First, choose $j \in\{0, \ldots, i\}$ with probability $\frac{1}{i+1}$. Second, choose $a \in A_{j}$ with probability $\frac{1}{p^{j}}$. Third, we choose an ordinal $\alpha \in I\left(v_{i}(a)\right)$ with probability $\mu_{v_{i}(a)}(\alpha)$. Finally, we select any subgroup $A$ of $A_{j}$ fairly with equal probability $p\left(A_{j}\right)$.

We now distinguish between several cases. If $\left(A_{i}, v_{i}, a, \alpha\right)$ realizes the non-critical case of the proof of Lemma 3.2, then we construct $\left(A_{i+1}, v_{i+1}\right)$ with $A_{i+1}=\left\langle A_{i}, p_{i}\right\rangle$, $p p_{i}=a$ and $v_{i+1}\left(p_{i}\right)=\alpha$ as described there. In particular, $v_{i+1}\left(x+m p_{i}\right)=$ $\min \left\{v_{i}(x), \alpha\right\}$ for all $x \in A_{i}$ and $0<m<p$. Then $f_{A_{i+1}, v_{i+1}} \leq f$.

Now assume that $\left(A_{i}, v_{i}, a, \alpha\right)$ realizes the critical case of the proof of Lemma 3.2 . 
First, in case all elements $x^{\prime} \in A_{i}$ as in condition (3.1) satisfy $p x^{\prime}+a=0$, then we construct $A_{i+1}=\left\langle A_{i}, p_{i}\right\rangle$ with universe $\left\{0, \ldots, p^{i+1}-1\right\}, p p_{i}=a$ and $f_{A_{i+1}, v_{i+1}} \leq f$ in an arbitrary way (e.g., by any predetermined process). This is clearly possible by the finiteness of $A_{i}$ and the assumption that $\lambda(f) \geq \omega$.

Second, assume there is $x^{\prime} \in A_{i}$ as in condition (3.1) with $p x^{\prime}+a \neq 0$. Then we construct $\left(A_{i+1}, v_{i+1}\right)$ with $A_{i+1}=\left\langle A_{i}, p_{i}\right\rangle, p p_{i}=a$ and $v_{i+1}\left(p_{i}\right)=\alpha$ as described in the proof of Lemma 3.2 . We restate the main steps.

We choose, say, a minimal element $x^{\prime} \in A_{i}$ (with respect to the natural order of $\left.A_{i}\right)$ with $v_{i}\left(x^{\prime}\right)=\alpha, v_{i}\left(p x^{\prime}+a\right) \geq \alpha+2, p x^{\prime}+a \neq 0$, and $x^{\prime} \notin A$ if possible. We use $x^{\prime}$ to extend the valuation to $A_{i+1}$. If $x \neq m x^{\prime}$, then put $v_{i+1}\left(x+m p_{i}\right)=$ $\min \left\{v_{i}(x), \alpha\right\}$, and if $x=m x^{\prime}$ and $v_{i}\left(p x^{\prime}+a\right)=\gamma+1$ is a successor ordinal, then put $v_{i+1}\left(x+m p_{i}\right)=\gamma$, and finally, if $v_{i}\left(p x^{\prime}+a\right)$ is a limit ordinal, then $v_{i+1}\left(x+m p_{i}\right)=\alpha+n$ for some suitable $n \in \mathbb{N}\left(\right.$ with $\alpha+n \notin v_{i}\left(A_{i}\right)$ and $\left.f(\alpha+n) \neq 0\right)$. Note that by our assumption $p x^{\prime}+a \neq 0$, Case $\mathrm{C}$ cannot occur. Hence we obtain $f_{A_{i+1}, v_{i+1}} \leq f$ as needed.

Finally, let $(G,+, v)=\bigcup_{i \in \mathbb{N}_{0}}\left(A_{i},+, v_{i}\right)$. As noted before, $f_{G, v} \leq f$ as required.

4.2. The Main Theorem. We want to establish the following.

Theorem 4.1. Let $f$ be a countable Ulm-sequence of length $\lambda \geq \omega$ and $\left(\mu_{\kappa}\right)_{\kappa \leq \lambda}$ be a sequence of discrete probability measures as above. Then the construction (Section 4.1) produces with probability 1 a group $(G,+, v)$ with $v=h_{G}$, the height valuation of $G$ and $f_{G}=f$.

Proof. We show that with probability 1 , the group $(G,+, v)$ constructed realizes all one-point $f$-extensions of finite subgroups. Then the result follows from Lemma 3.4 .

Since $\lambda$ is countable, there are only a countable number of one-point $f$-extensions of finite subgroups of $(G,+, v)$. Note that the intersection of countably many events of probability 1 again has probability 1 . Hence it suffices to consider an arbitrary fixed one-point $f$-extension $(B, v)$ of a finite subgroup $(A, v)$ of $(G,+, v)$ and to show that it is realized almost surely.

By assumption there is $y \in B \backslash A$ such that $B=\langle A, y\rangle$ and $a=p y \in A$. We may assume that $v(y)=\sup v(B \backslash A)$ and $v(p y) \geq v\left(p y^{\prime}\right)$ for each $y^{\prime} \in B \backslash A$ with $v(y)=v\left(y^{\prime}\right)$. Put $\alpha=v(y)$. As we showed in the proof of Lemma 3.3. now $v(a) \geq \alpha+2$ implies $f_{A}(\alpha)<f_{B}(\alpha) \leq f(\alpha)$, so $f(\alpha) \neq 0$ and hence $\alpha \in I(v(a))$.

Since $A$ is finite, there is $j \in \mathbb{N}_{0}$ with $A \subseteq A_{j}$. Choose any $i \geq j$. We wish to determine a lower bound for the probability that there is a valuated embedding $\varphi: B \hookrightarrow A_{i+1}$ with $\varphi\left\lceil A=\mathrm{id}_{A}\right.$.

The probability that in the first step of our construction of $A_{i+1}$ we chose this number $j$ is $\frac{1}{i+1}$. Next, the probability that in the second step we chose precisely this element $a$ is $\frac{1}{p^{j}}$. The probability that we determine $v\left(p_{i}\right)$ to be exactly $\alpha=v(y)$ is $\mu_{v(a)}(\alpha)$. We note that $\mu_{v(a)}(\alpha) \neq 0$. The probability that we choose $A$ as our subgroup of $A_{j}$ is $p\left(A_{j}\right)$. In total, the probability of realizing all these conditions is $\frac{1}{i+1} \frac{1}{p^{j}} \mu_{v(a)}(\alpha) p\left(A_{j}\right)$. A lower bound is $\frac{1}{i+1} \epsilon$ with $\epsilon=\frac{1}{p^{j}} \mu_{v(a)}(\alpha) p\left(A_{j}\right)$ which depends on $j$ and the structure of the one-point $f$-extension $(A, v) \subseteq(B, v)$ but not on $i$.

Now we consider the amalgamation diagram $A \subseteq B, A \subseteq A_{i}$, the proof of Lemma 3.3 , and the construction of $\left(A_{i+1}, v_{i+1}\right)$. If the quadruple $\left(A_{i}, v_{i}, a, \alpha\right)$ realizes the non-critical case of the proof of Lemma 3.2 by the proof of Lemma 3.3 we obtain a valuated embedding $\varphi: B \longrightarrow A_{i+1}$ with $\varphi\left\lceil A=\operatorname{id}_{A}\right.$. Now assume that 
$\left(A_{i}, v_{i}, a, \alpha\right)$ realizes the critical case of the proof of Lemma 3.2. Following the proof of Lemma 3.3 we distinguish between several cases. In all cases, there is $x^{\prime} \in A_{i} \backslash A$ with $v_{i}\left(x^{\prime}\right)=\alpha$ and $v_{i}\left(p x^{\prime}+a\right) \geq \alpha+2$. In case $p x^{\prime}+a=0$, we obtain a valuated embedding $\varphi: B \longrightarrow A_{i}$ with $\varphi \mid A=\mathrm{id}_{A}$. In case $p x^{\prime}+a \neq 0$, we constructed $A_{i+1}=\left\langle A_{i}, p_{i}\right\rangle$ with $p p_{i}=a$ and the valuation $v_{i+1}$ on $A_{i+1}$ as described in the proof of Lemma 3.2. Then there is a valuated embedding $\varphi: B \longrightarrow A_{i+1}$ with $\varphi\left\lceil A=\mathrm{id}_{A}\right.$. Hence, since $A_{i} \subseteq A_{i+1}$, in any case at least with probability $\frac{\epsilon}{i+1}$, there is a valuated embedding $\varphi: B \hookrightarrow A_{i+1}$, with $\varphi\left\lceil A=\operatorname{id}_{A}\right.$.

Thus the probability that there is no embedding $\varphi: B \hookrightarrow A_{i+1}$ with $\varphi\left\lceil A=\operatorname{id}_{A}\right.$ is at most $1-\frac{\epsilon}{i+1}$. Consequently, the probability that for no $i \geq j$ there is an embedding $\varphi: B \hookrightarrow A_{i+1}$ with $\varphi\left\lceil A=\operatorname{id}_{A}\right.$ is at most $\prod_{i \geq j}\left(1-\frac{\epsilon}{i+1}\right)=0$ by a standard result of analysis on infinite products, since $\sum_{i \geq j} \frac{\epsilon}{i+1}=\infty$. Therefore the one-point $f$-extension $(A, v) \subseteq(B, v)$ is realized in $(G,+, v)$ by our construction with probability 1.

We note that this construction leaves us certain freedoms. For example, when we construct $A_{i+1}$ given $A_{i}$ and the choices of $a$ and $\alpha$, there are in general several ways to define the addition on the set $A_{i+1}$ in order to obtain a valuated abelian $p$ group having $A_{i}$ as subgroup as prescribed. Here, for simplicity we take the natural addition given by $\left(x+m p_{i}\right)+\left(y+n p_{i}\right)=(x+y)+(m+n) p_{i}$ for all $x, y \in A_{i}$ and $0 \leq m, n<p$, and with $p p_{i}=a$.

Any other construction of the addition of $A_{i+1}$ (subject to the given restrictions) would work equally well, but it would determine in Section 5 the conditional probabilities described there and thereby lead to a different probability space $\Omega$.

\section{The Probability $\operatorname{Space}(\Omega, \mathfrak{A}, \mu)$ For Theorem 4.1}

For the reader interested in a formal description we give an exact definition of the probability space $(\Omega, \mathfrak{A}, \mu)$ related to Theorem 4.1. We follow 9 ; see also 11 for basic notions.

Let $f$ be a countable Ulm-sequence of length $\lambda \geq \omega$ and $\left(\mu_{k}\right)_{k \leq \lambda}$ a sequence of discrete probability measures as in Section 4.1. For $n \in \mathbb{N}$ again let $A_{n}=$ $\left\{0, \ldots, p^{n}-1\right\}$. Our construction produces a valuated $p$-group $G=(\mathbb{N},+, v)$ with subgroups $A_{n} \subseteq G$ for all $n \in \mathbb{N}$ and Ulm-invariant $f_{G, v} \leq f$. Therefore let $\Omega$ denote the set of all valuated $p$-groups $G=\left(\mathbb{N}_{0},+, v\right)$ on $\mathbb{N}_{0}$ with Ulm-invariant $f_{G, v} \leq f$ such that $A_{n}$ is a subgroup of $G$, for all $n \in \mathbb{N}$. Similarly, for each element $n \in \mathbb{N}_{0}$, let $\Omega_{n}$ be the set of all valuated $p$-groups on $A_{n}$, i.e. all pairs of relations $(+, v)$ on $A_{n}$ such that $\left(A_{n},+, v\right)$ is a valuated abelian $p$-group, and $A_{m} \subseteq A_{n}$ is a valuated subgroup for all $m \leq n$.

We want to define a discrete probability measure $\mu_{n}$ on each $\Omega_{n}\left(n \in \mathbb{N}_{0}\right)$. Let $n \in \mathbb{N}_{0}$ and assume that we have defined $\mu_{n}$ on $\Omega_{n}$. If $(+, v)_{n} \in \Omega_{n}$, then we want to extend the valuated $p$-group $\left(A_{n},(+, v)_{n}\right)$ to $\left(A_{n+1},(+, v)_{n+1}\right) \in \Omega_{n+1}$. Our probabilistic procedure (Section 4.1) thus determines the intuitive conditional probability $P\left((+, v)_{n+1} \mid(+, v)_{n}\right)$ of $(+, v)_{n+1}$ given its restriction $(+, v)_{n}$ on $A_{n}$. Putting $\mu_{0}\left((+, v)_{0}\right)=1$ and, inductively, $\mu_{n+1}\left((+, v)_{n+1}\right)=P\left((+, v)_{n+1} \mid(+, v)_{n}\right)$. $\mu_{n}\left((+, v)_{n}\right)$ for any $(+, v)_{n+1} \in \Omega_{n+1}$ extending $(+, v)_{n}$, we obtain a discrete measure $\mu_{n+1}$ on $\Omega_{n+1}$ such that $P\left((+, v)_{n+1} \mid(+, v)_{n}\right)$ becomes the exact conditional probability of $(+, v)_{n+1}$, given its restriction $(+, v)_{n}$ to $A_{n}$. Inductively, we get 
$\mu_{n+1}\left((+, v)_{n+1}\right)=\prod_{0 \leq m \leq n} P\left((+, v)_{m+1} \mid(+, v)_{m}\right)$ and $(+, v)_{n+1}\left\lceil A_{m}=(+, v)_{m}\right.$ for all $0 \leq m \leq n+1$.

Finally we want to extend $\mu_{n}(n \in \mathbb{N})$ to $\Omega$. There is a natural mapping $g_{n}$ : $\Omega_{n+1} \longrightarrow \Omega_{n}$, which is the restriction just described. Given any $(+, v)_{n} \in \Omega_{n}$, then

$$
\sum\left\{P\left((+, v)_{n+1} \mid(+, v)_{n}\right):(+, v)_{n+1} \in \Omega_{n+1},(+, v)_{n+1}\left\lceil A_{n}=(+, v)_{n}\right\}=1\right.
$$

and thus $\left.\mu_{n}\left((+, v)_{n}\right)=\sum\left\{\mu_{n+1}\left((+, v)_{n+1}\right): \mu_{n+1} \in \Omega_{n+1}, g_{n}\left((+, v)_{n+1}\right)=(+, v)_{n}\right)\right\}$. Hence we obtain a projective system whose projective limit is $\Omega$.

If $(A,+, v) \in \Omega$ and $n \in \mathbb{N}$, let $\pi_{n}(+, v)=(+, v) \uparrow A_{n}$. Then the Borel $\sigma$-algebra $\mathfrak{A}$ on the projective limit is generated by $\left.\left\{\pi^{-1}(+, v)_{n}\right) \mid n \in \mathbb{N},(+, v)_{n} \in \Omega_{n}\right\}$. The Prokhorov extension theorem (see [1, Section 3.1]) asserts that there exists a unique probability measure $\mu$ on $\mathfrak{A}$ satisfying $\left.\mu\left(\pi^{-1}(+, v)_{n}\right)=\mu_{n}\left((+, v)_{n}\right)\right)$ for all $n \in \mathbb{N}$ and $(+, v)_{n} \in \Omega_{n}$. Here $\pi^{-1}(+, v)_{n}$ are the relations of those valuated $p$-groups in $\Omega$ to which $(+, v)_{n}$ can be extended; thus the probability of this generating set in $\mathfrak{A}$ equals $\mu\left((+, v)_{n}\right)$, the probability of having constructed $\left(A_{n},(+, v)_{n}\right)$ in finitely many steps. Therefore the probability space $(\Omega, \mathfrak{A}, \mu)$ is the space of all possible valuated abelian $p$-groups that we consider.

Now we formulate our main result (Theorem 4.1) as follows.

Theorem 5.1. Let $f$ be a countable Ulm-sequence of length $\lambda \geq \omega$ and $\left(\mu_{\kappa}\right)_{\kappa \leq \lambda}$ be a sequence of discrete probability measures as above. In the probability space $(\Omega, \mathfrak{A}, \mu)$ for $f$ as above, the set $\left\{G \in \Omega \mid f_{G}=f\right\}$ has measure 1 .

We want to apply the theorem to abelian groups $G$ with the cancellation property. This is the case if, for any abelian groups $H$ and $K, G \oplus H \cong G \oplus K$ implies $H \cong K$. We derive the following amusing corollary

Corollary 5.2. Let $f$ be a countable Ulm-sequence of length $\lambda>\omega$ and at least one infinite value. In the probability space $(\Omega, \mathfrak{A}, \mu)$ for $f$ the set $\{G \in \Omega$ | $G$ has the cancellation property\} has measure 0 .

Hence countable abelian $p$-groups with the cancellation property, even if we permit just a few more with some infinite Ulm-invariant, are rare. This is the probabilistic implication of Crawley's theorem:

Proof. By Theorem [5.1, the set $\left\{G \in \Omega \mid f_{G}=f\right\}$ has measure 1. Hence $\{G \in \Omega \mid$ $\left.f_{G}<f\right\}$ has measure 0 and contains the set $\left\{G \in \Omega \mid f_{G} \leq f, f_{G}\right.$ is finite $\}$. By Crawley [4] the latter set characterizes the Ulm-invariants $\leq f$ of groups $G$ with the cancellation property; see also Fuchs [13, p. 67, Exercise 6].

(Clearly, if we restrict $f$ to finite Ulm-invariants and let $(\Omega, \mathfrak{A}, \mu)$ be its probability space, then all groups from $\Omega$ have the cancellation property.) As a further example, we note that as a consequence of Theorem 4.1, the two subsets $A=\left\{G \in \Omega \mid f_{G, v}=f\right\}$ and $B=\left\{G \in \Omega \mid v_{G}=h_{G}\right\}$ both have measure 1, but are in general not contained in each other. Indeed, any $G \in \Omega$ with $v_{G}=h_{G}$ and $f_{G}<f$ satisfies $G \in B \backslash A$. Conversely, choose an infinite reduced valuated $p$-group $(G, v)$ with $v \neq h_{G}$ and put $f=f_{G, v}$. Then an isomorphic copy $G^{\prime}$ of $G$ belongs to $\Omega$; hence $G^{\prime} \in A \backslash B$.

We note that given the Ulm-sequence $f$, the set $\Omega$ can also be endowed, in a natural way, with a metric yielding a complete metric space $\Omega$ of abelian $p$-groups on $\mathbb{N}$. Then the collection of all groups $G \in \Omega$ with $f_{G}=f$ can be shown to be 
co-meagre (the complement of a meagre set), hence again 'large', now in the topological sense. For background see Cameron [3], for similar results on topological spaces of Scott domains see [8], and for related recent results see Goldstern [15].

\section{ACKNOWLEDGEMENT}

The authors would like to thank the anonymous referee, whose careful remarks have resulted in improvements to this paper.

\section{REFERENCES}

[1] S. Abbes and K. Keimel, Projective topology on bifinite domains and applications, Theoretical Computer Science, 365 (2006), 171 - 183. MR2267074 (2008h:68079)

[2] A. Blass and G. Braun, Random orders and gambler's ruin, Electronic Journal of Combinatorics 12 (2005), Research paper 23. MR2134186 (2005m:05008)

[3] P. J. Cameron, Oligomorphic Permutation Groups, Cambridge Univ. Press, 1990. MR:1066691 (92f:20002)

[4] P. Crawley, The cancellation of torsion abelian groups in direct sums, Journal of Algebra 2 (1965), 432 - 442. MR0188293 (32:5732)

[5] M. Droste, Universal homogeneous causal sets, Journal of Math. Physics 46 (2005), 122503. MR2194024 (2007f:83039)

[6] M. Droste and G.-Q. Zhang, Random event structures, International Journal of Software and Informatics 2 (2008), $77-88$.

[7] M. Droste and R. Göbel, A categorical theorem on universal objects and its application in abelian group theory and computer science, Contemporary Math. 131, Part 3, Amer. Math. Soc., 1992, 49 - 74. MR1175872 (93g:18003)

[8] M. Droste and D. Kuske, On random relational structures, Journal of Combinatorial Theory, Series A 1022 (2003), 241 - 254. MR1979531 (2005a:03064)

[9] M. Droste and D. Kuske, Almost every domain is universal, 23rd Conf. on the Mathematical Foundations of Programming Semantics (MFPS), Electronic Notes in Theoretical Computer Science 173 (2007) $104-119$.

[10] P. Erdős and A. Rényi, Asymmetric graphs, Acta Math. Acad. Sci. Hungar. 14 (1963), 295 315. MR0156334 (27:6258)

[11] P. Erdős and J. Spencer, Probabilistic Methods in Combinatorics, Probability and Mathematical Statistics, Academic Press, New York, 1974. MR0382007 (52:2895)

[12] R. Fraïssé, Sur l'extension aux relations de quelques propriétés des ordres, Ann. Sci. École Norm. Sup. (3) 71 (1954), 363 - 388. MR0069239 (16:1006b)

[13] L. Fuchs, Infinite Abelian Groups, Vols. 1 and 2, Academic Press, New York, 1970, 1973. MR0255673 (41:333), MR0349869 (50:2362)

[14] R. Göbel, Helmut Ulm, his work and its impact on recent mathematics, Contemporary Mathematics 87, Amer. Math. Soc., 1989, 1 - 10. MR.995259 (90c:01035)

[15] M. Goldstern, The typical countable algebra, pp. 57-71 in: Contributions to General Algebra 18 (eds. G. Dorfer, G. Eigenthaler, H. Kautschitsch, W. More, W.B. Müller), Verlag Johannes Heyn, Klagenfurt, 2008. MR2407575 (2010g:08010)

[16] W. Hodges, Model Theory, Cambridge University Press, Cambridge, 1993. MR:1221741 (94e:03002)

[17] R. Hunter, F. Richman, and E. Walker, Existence theorems for Warfield groups, Trans. Amer. Math. Soc. 235 (1978), 345 - 362. MR0473044 (57:12723)

[18] B. Jónsson, Homogeneous universal relational systems, Math. Scand. 8 (1960), 137 - 142. MR0125021 (23:A2328)

[19] R. Mines, F. Richman, and W. Ruitenberg, A Course in Constructive Algebra, Universitext, Springer-Verlag, Berlin, 1988. MR.919949 (89d:03066)

[20] F. Richman, The constructive theory of countable abelian p-groups, Pacific Journ. Math. 45 (1973), 621 - 637. MR0344088 (49:8828)

[21] F. Richman, A guide to valuated groups, pp. 73-86, in Abelian Group Theory, Lecture Notes in Math. 616, Springer, Berlin-Heidelberg, 1977. MR0480779(58:929) 
[22] F. Richman and E. Walker, Valuated groups, Journal of Algebra 56 (1979), 145 - 167. MR.527162 (80k:20053)

[23] H. Ulm, Zur Theorie der abzählbar-unendlichen Abelschen Gruppen, Math. Annalen 107 (1933), $774-803$. MR 1512826

[24] L. Zippin, Countable torsion groups, Ann. of Math. (2) 36 (1935), 86 - 99. MR1503210

Institute of Computer Science, Universität Leipzig, PF 100920, 04009 Leipzig, GerMANY

E-mail address: droste@informatik.uni-leipzig.de

Fakultät für Mathematik, Universität Duisburg-Essen, Campus Essen, 45117 Essen, GERMANY

E-mail address: ruediger.goebel@uni-due.de 